\title{
Addressing the need to diagnose and manage Pediatric Delirium - A Clinical Pathway
}

Gabrielle Silver, MD, Associate Clinical Professor, NYP Weill Cornell Medical College Julia Kearney, MD, Associate Professor, Memorial Sloane Kettering Center

\section{Objective}

Consider the use of a Clinical Pathway to guide recognition and management of pediatric delirium Collaboration between specialties and a Delirium Clinical Pathway

Pediatric Delirium is increasingly acknowledged to be highly prevalent, about $50 \%$ of critically ill youth. This non-traumatic brain injury or final common pathway of CNS disruption by illness, medications, and other toxic metabolic stressors is associated with concerning short and long-term outcomes. The development of screening tools, new approaches to sedation, and management of critically ill children has provided new opportunities for improving care. PaCC generated screening and intervention pathways for pediatric delirium that utilizes validated tools and evidence based recommendations for intervention where available. Implementation of these pathways informs and emerges from collaboration between medical personnel.

\section{Method}

Emerging research identifying prevalence of pediatric delirium and associated increased morbidity and mortality is exposing the vulnerability of the brain in our youngest patients. There is a need for health care professionals in the ER, critical care, and those working with vulnerable populations to have guidance in recognition, prevention and management of Pediatric Delirium to improve collaboration and improve care. These Clinical Pathways developed by expert consensus, using evidence base can help institutions develop and implement necessary, structured screening, prevention and management systems. This pathway was developed by a team of consultation child and adolescent psychiatry experts incorporating stakeholder feedback.

\section{Results and Conclusion}

Collaborative efforts between healthcare providers with the guidance of well designed, easy to use and widely accepted clinical pathway for delirium recognition, prevention and management may greatly facilitate consensus and evidence based approach to improve outcomes for pediatric patients. Given that Pediatric delirium is an important contributor to morbidity and mortality in Pediatrics a need for approaches that guide clinical and research efforts is needed.

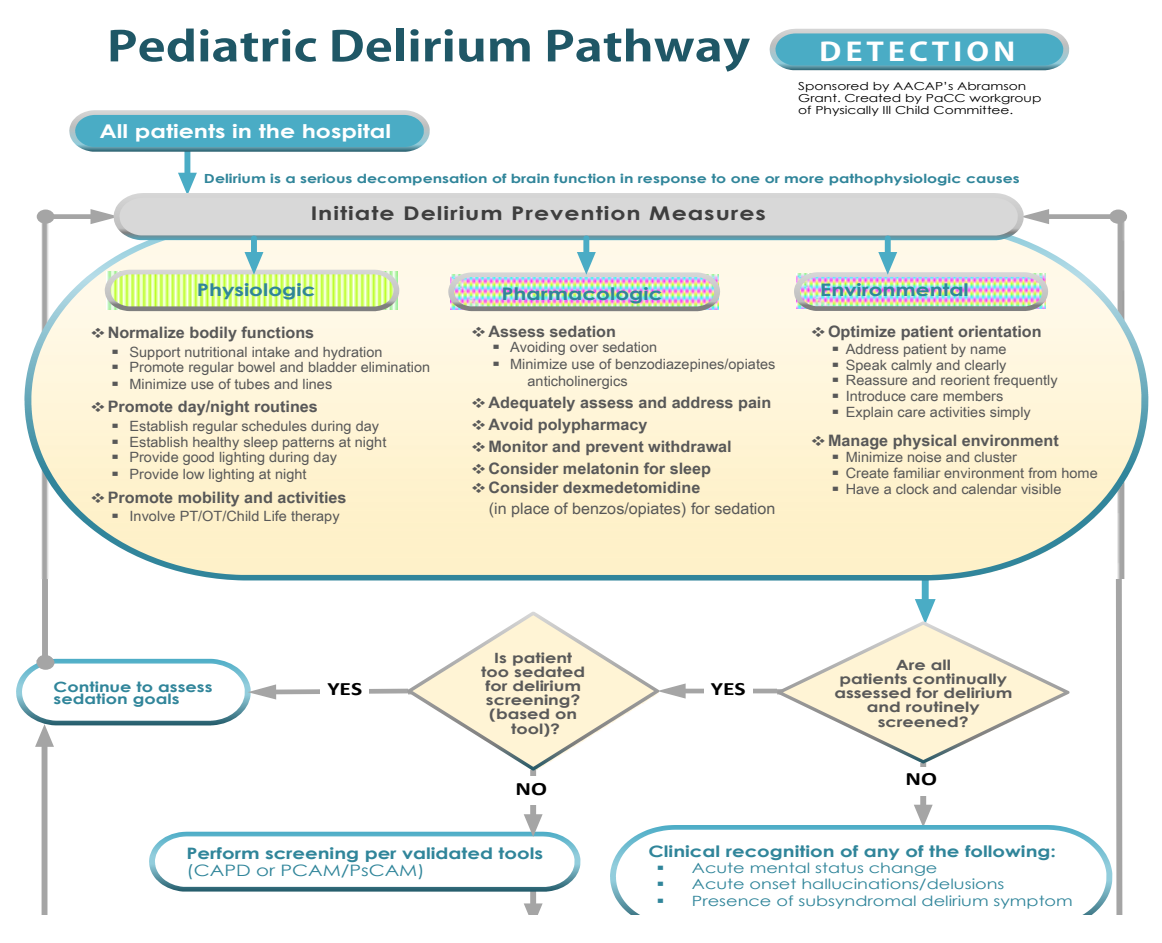

Traube C, Silver G, Gerber LM, et al. Delirium and Mortality in Critically III Children: Epidemiology and Outcomes of Pediatric Delirium. Crit Care Med. 2017;45(5):891-898.

Traube C, Silver G, Kearney J, et al. Cornell Assessment of Pediatric Delirium: a valid, rapid, observational tool for screening delirium in the PICU*. Crit Care Med. 2014;42(3):656-663.

Smith HA, Boyd J, Fuchs DC, et al. Diagnosing delirium in critically ill children: Validity and reliability of the Pediatric Confusion Assessment Method for the Intensive Care Unit. Crit Care Med. 2011;39(1):150-157. Simone S, Edwards S, Lardieri A, et al. Implementation of an ICU Bundle: An Interprofessional Quality Improvement Project to Enhance Delirium Management and Monitor Delirium Prevalence in a Single PICU. Pediatr Crit Care Med. 2017;18(6):531-540.

Smith HA, Gangopadhyay M, Goben CM, et al. The Preschool Confusion Assessment Method for the ICU: Valid and Reliable Delirium Monitoring for Critically III Infants and Children. Crit Care Med. 2016;44(3):592-600.

Kawai Y, Weatherhead JR, Traube C, et al. Quality Improvement Initiative to Reduce Pediatric Intensive Care Unit Noise Pollution With the Use of a Pediatric Delirium Bundle. J Intensive Care Med. 2017:885066617728030.

Traube C, Silver G, Kearney J, et al. Cornell Assessment of Pediatric Delirium: A Valid, Rapid, Observational Tool for Screening Delirium in the PICU. Crit Care Med. 2013.

Silver G, Kearney J, Traube C, Hertzig M. Delirium screening anchored in child development: The Cornell Assessment for Pediatric Delirium. Palliat Support Care. 2015;13(4):1005-1011
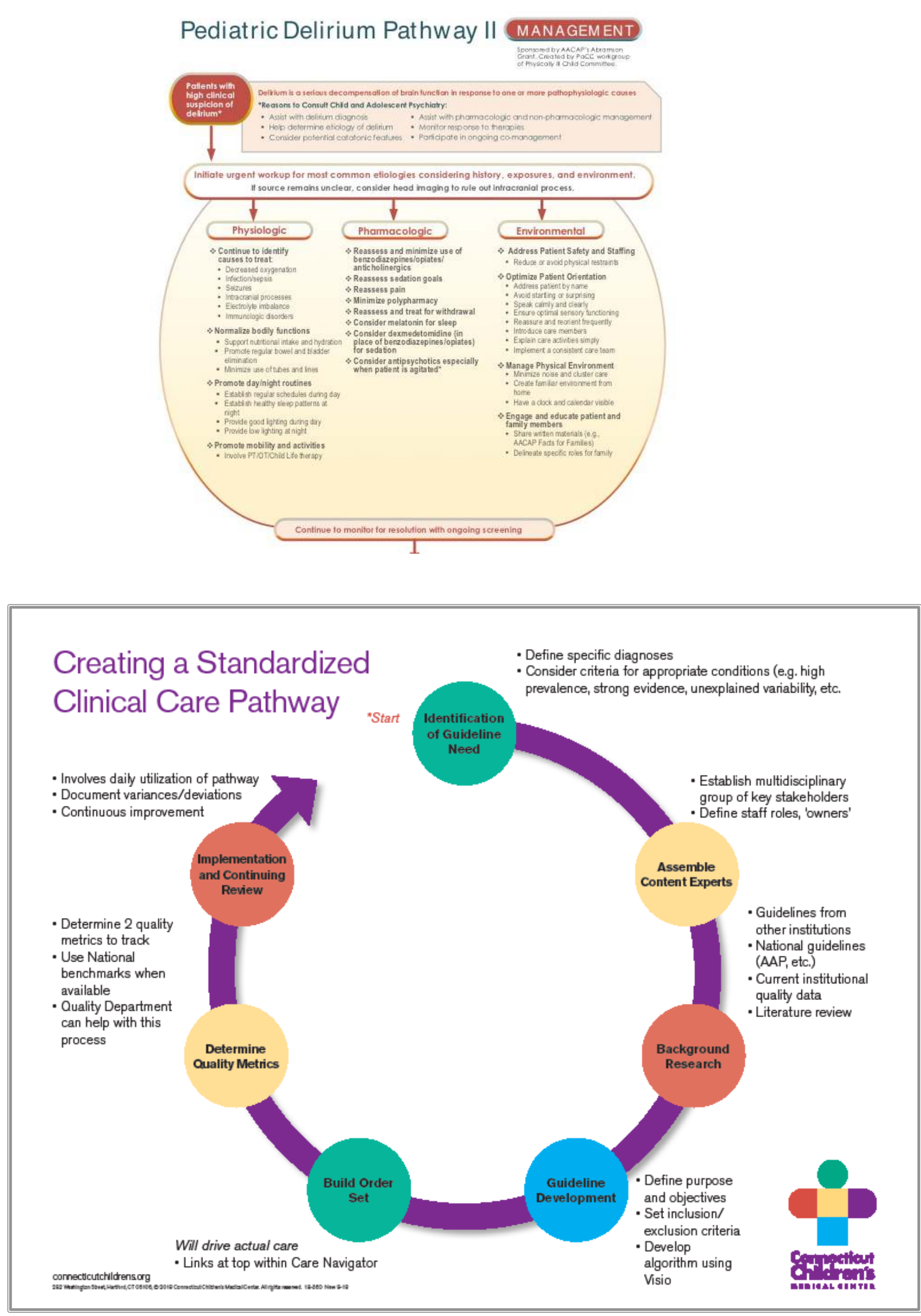